Original Research Paper

\title{
Exploratory Analysis of Rainfall Occurrence in South Sulawesi Region Using Spatial Point Process
}

\author{
${ }^{1}$ Nurtiti Sunusi, ${ }^{1}$ Erna Tri Herdiani, ${ }^{1}$ Fachrul Nawawi, ${ }^{2}$ Giarno \\ ${ }^{1}$ Department of Mathematics, Faculty of Mathematics and Natural Sciences, Hasanuddin University, Indonesia \\ ${ }^{2}$ Department of Meteorology, Climatology and Geophysics, Paotere, Makassar, Indonesia
}

\author{
Article history \\ Received: 16-06-2015 \\ Revised: 26-10-2015 \\ Accepted: 17-11-2015 \\ Corresponding Author: \\ Nurtiti Sunusi \\ Department of Mathematics, \\ Faculty of Mathematics and \\ Natural Sciences, Hasanuddin \\ University, Indonesia \\ Email: ntitisanusi@gmail.com
}

\begin{abstract}
This paper study the probability of rainfall occurrence in round year in different segment in South Sulawesi region. In this research, rainfall occurrence in round year described by one line which has divided into 12 months. Each one of those months is assumed that the probability of a rainfall follow a homogeneous Poisson distribution. To modeling the rainfall occurrence in round year, a spatial point process is used. The parameter of the model is estimated by Seemingly Unrelated Regression (SUR) method and Ordinary Least Square (OLS) method with assume that two stations have a correlation in residual model. Results of case study on monthly rainfall data indicate that when the residual correlation (autocorrelation) on all models is weakly and not significant. Thus, it has not good enough to use the SUR method for increase efficiency compared with the OLS method. Moreover, results of the parameter estimation of the model for two selected stations (Paotere and Mandai) showed that the SUR method is more representative than the OLS method.
\end{abstract}

Keywords: Spatial Point Process, Ordinary Least Square, Seemingly Unrelated Regression

\section{Introduction}

Forecasting is a science to predict events in the future which can be done by using past data into a mathematical model to predict the future of data. In forecasting, data that has dependencies on time is used. It was taken in a certain time within the same time interval. Furthermore, the influence of the location (space) is taken into account, or in this case knows as the space-time data. Data is measured against several observation sites, so in addition to having dependencies on time, the data space time also have dependencies on space. Rainfall phenomena are occurs in random and has dependencies on time. Spatial Point Process is a stochastic model that was built on the site of a phenomenon $\left\{S_{i}\right\}$ on the set X. One of the simplest models of point process is Poisson process. There is extensive literature on the use of Poisson cluster processes in the stochastic modeling of rainfall, stemming largely from (Rodriguez-Iturbe et al., 1987; Onof et al., 2000; Cameron et al., 2000). Rainfall modeling can generally be classified into four categories (Onof et al., 2000): (1) Meteorological models involving complex sets of differential equations representing the physical processes controlling precipitation and other weather variables; (2) stochastic multiscale models describing the spatial evolution of the rainfall process independently of scale; (3) statistical models which can allow for the modeling of trends; and (4) stochastic process.

Some previous researchers have done a study on parameter estimation of the model, among others (Alaba et al., 2010; Vasco, 2012; Atanlogun et al., 2014). Alaba et al. (2010) showed the efficiency of SUR method compared with OLS method. In other research, Vasco (2012) use SUR to predict the carcass composition of Lambs. Furthermore, Atanlogun et al. (2014) studied the comparison between OLS method and SUR method. The result of analysis shows that OLS and SUR methods have a standard error and coefficient value in simultaneous equation.

In recent years, the occurrence of rain in parts of Indonesia is difficult to predict because of the erratic appearance. Rainfall in parts of Indonesia almost evenly, but in some areas there is a difference in terms of the level/intensity of rainfall. Rainfall in Indonesia are generally within the normal range, but in several areas including South Sulawesi and surrounding areas, the rainfall will exceed the normal limits on certain months. Therefore, these phenomena can be modeled using a model point process which is reviewed based on the location that has the level of rainfall exceeds 
normal limits. To estimate parameter model, SUR and OLS method is used.

This paper will compare the parameter estimation method of point process model between SUR and OLS. Both of methods are applied to rainfall data of South Sulawesi region during 5 years. The rainfall data used in this study is taken from www.ogimet.com.

Let rainfall in an area assumed to be constant within a period of one year, then the phenomenon of rain events Stationary approximated by a Poisson process. Model of stationary Poisson is (Daley and Verre Jones, 2003):

$$
\begin{aligned}
& P\left\{N\left(m_{i}, m_{i+1}\right]=n_{i}, i=0, \ldots, M B\right\} \\
& =\prod_{i=1}^{M B} \frac{\lambda\left(b_{i}, b+i+1\right)}{n_{i}} n_{i}^{\lambda\left(b_{i}, b_{i+1}\right)}
\end{aligned}
$$

where, in the equation in accordance with the annual rainfall phenomenon which includes 3 important things, namely:

- The amount of rainfall in each month $\left(m_{i}, m_{i+1}\right]$ throughout a given year is Poisson distributed

- The number of rainfall with very high intensity in every disjoint month is a random variable that is independent

- Stationary distributed: Specific for each month means only depend on the month $b_{i+1}, b_{i}$ of the total months of the year

\section{Notation and Assumptions}

Indonesian territory located in the tropics affect the amount of rainfall received. The average rainfall in Indonesia is $2.000 \mathrm{~mm} /$ year, or about $150-170 \mathrm{~mm} / \mathrm{month}$. The category of medium rainfall is $100-300 \mathrm{~mm} / \mathrm{month}$. The occurrence of erratic rains throughout the year but the chances of rain events with a category of rainfall intensity with very high category $(>400 \mathrm{~mm} / \mathrm{month})$ are very small. Therefore, the incidence of rain with very high intensity can be approximated to the Poisson model. Rainfall in Indonesia are generally within the normal range, but in several areas including South Sulawesi and surrounding areas, the rainfall will exceed the normal limits on certain months. Therefore, these phenomena can be modeled using a model point process which is reviewed based on the location that has the level of rainfall exceeds normal limits. The model for a nearby location is assumed to have cross-correlated error.

In this study, the phenomenon in question is a rain event and the set $X$ is a subset of $R$, because every month will be represented by intervals of real numbers. In this study, the time interval of one year consider as a line. It was divided into twelve months and each month drawn into a stationary Poisson process.

\section{SUR and OLS Method}

SUR is a generalization of a linear regression model that consists of several regression equations, each having its own dependent variable and potentially different sets of exogenous explanatory variables. The model can be estimated equation-byequation using standard Ordinary Least Squares (OLS). Such estimates are consistent, however generally not as efficient as the SUR method, which amounts to feasible generalized least squares with a specific form of the variance-covariance matrix.

\section{Intensity of Rainfall Occurrence}

Rainfall event within one month $b_{i}$ is the time of the beginning of each month, $M B$ is the total months and lambda is a parameter of the Poisson distribution.

Intensity of rainfall in one month was defined as density, which in this study is the average rainfall per month. This is consistent with the parameters of the Poisson distribution, as follows:

$$
\lambda=\lim _{\left(b_{i}, b_{i+1}\right] \rightarrow 0} \frac{E\left[N\left(b_{i}, b_{i+1}\right]\right]}{\left(b_{i}, b_{i+1}\right)}
$$

Consider that the intensity $\lambda$ in each month $\left(b_{i}, b_{i+1}\right]$ tends to a constant value. The value of $\lambda$ is a constant can be interpreted as the average rate or the average density of events in the process. A constant $\lambda$ value also means that the rain event is an example of a complete spatial randomness, or in other words that the whole year $\left(b_{i}, b_{i+1}\right]$ have the same opportunities to the occurrence of rain.

Mean $\mu\left(b_{i}, b_{i+1}\right]$ and Variance $V\left(b_{i}, b_{i+1}\right]$ of the number of rain which occur in $\left(b_{i}, b_{i+1}\right]$ is defined by:

$\mu\left(b_{i}, b_{i+1}\right]=\lambda\left(b_{i}, b_{i+1}\right]=V\left(b_{i}, b_{i+1}\right]$

Equation 3 shows that process that occurs in one month is homogeneous-stationary Poisson process. To review the process that occurs in the whole month of the year is used non homogeneous Poisson process, where every month has a function of spatial location. In other words, the intensity of a non homogeneous Poisson process can vary. From Equation 1, we obtained quantity as follows:

$\lambda_{i}=\int_{b_{i}}^{b_{i+1}} \lambda(x) d x$

Based on Equation 4, we know that Poisson parameter $\lambda$ is constant in every year but it is different for each month.

The months are prone to high intensity rainfall can be identified through the decomposition of the multiplication 
of the scene at the time of the point intensity rainfall events, in this case is the estimate of the intensity at each location of the overall chances of rain events. The time period is divided into day and night with the assumption that the rate of accidents between day and night was different. In order to obtain a Poisson distribution of rain intensity for non homogeneous case are as follows:

$\lambda_{j i}=\lambda_{j}(x) P_{\text {rain }} ; X \in\left(b_{i}, b_{i+1}\right], j=$ station $I$, station II

The overall intensity of the rain is the amount of rain per month divided by the total of rain event in a year. It is not relevant to the case in which the intensity of each month will be influenced by the amount of rainfall events that occurred in the previous month. Based on that, we need to be evaluated against $\lambda_{j i}$.

\section{Results}

Estimating Parameter of Spatial Point Process Model with SUR and OLS

To evaluate $\lambda_{j i}$ together in one system, we used models Seemingly Unrelated Regression (SUR). SUR models used to calculate the effect of the errors that are correlated in a system of equations.

All factors can be taken into account including variable proxy (whether vulnerable segments of the month as well as the number of rain). Then location was divided into two stations that each station modeled into a regression equation in which every equation has a parameter that can be found with the usual OLS method. Due to the correlation between the errors that occurs resulting parameters are obtained in theory does not possess Best Linear Unbiased Estimator (BLUE). To obtain the parameters that have the properties of both BLUE, the system can be expressed into a set of equations and estimated parameters in one settlement.

Station I:

$$
\begin{aligned}
& \lambda_{11}=X_{11}^{1} \beta_{1}^{1}+X_{12}^{1} \beta_{2}^{1}+\ldots+X_{112}^{1} \beta_{12}^{1}+\varepsilon_{11} \\
& \lambda_{12}=X_{21}^{1} \beta_{1}^{1}+X_{22}^{1} \beta_{2}^{1}+\ldots+X_{212}^{1} \beta_{12}^{1}+\varepsilon_{12} \\
& \vdots \\
& \lambda_{13}=X_{31}^{1} \beta_{1}^{1}+X_{32}^{1} \beta_{2}^{1}+\ldots+X_{312}^{1} \beta_{12}^{1}+\varepsilon_{13}
\end{aligned}
$$

\section{Station II:}

$$
\begin{aligned}
& \lambda_{21}=X_{11}^{1} \beta_{1}^{1}+X_{12}^{1} \beta_{2}^{1}+\ldots+X_{12}^{1} \beta_{12}^{1}+\varepsilon_{21} \\
& \lambda_{22}=X_{21}^{1} \beta_{1}^{1}+X_{22}^{1} \beta_{2}^{1}+\ldots+X_{212}^{1} \beta_{12}^{1}+\varepsilon_{22} \\
& \vdots \\
& \lambda_{25}=X_{51}^{1} \beta_{1}^{1}+X_{52}^{1} \beta_{2}^{1}+\ldots+X_{512}^{1} \beta_{12}^{1}+\varepsilon_{55}
\end{aligned}
$$

Equation 6 can be written as follows (Hill at al., 2012):

$$
\left[\begin{array}{c}
\lambda_{11} \\
\vdots \\
\lambda_{15} \\
\lambda_{21} \\
\vdots \\
\lambda_{25}
\end{array}\right]=\left[\begin{array}{cccccc}
X_{11}^{1} & \cdots & X_{112}^{1} & 0 & \cdots & 0 \\
\vdots & \ddots & \vdots & \vdots & \ddots & \vdots \\
X_{51}^{1} & \vdots & X_{512}^{1} & 0 & \cdots & 0 \\
0 & \ddots & 0 & X_{11}^{2} & \cdots & X_{112}^{2} \\
\vdots & \cdots & \vdots & \vdots & \cdots & \vdots \\
0 & \ddots & 0 & X_{51}^{2} & \cdots & X_{512}^{2}
\end{array}\right] x\left[\begin{array}{c}
\beta_{11}^{1} \\
\vdots \\
\beta_{112}^{1} \\
\beta_{11}^{2} \\
\vdots \\
\beta_{112}^{2}
\end{array}\right]+\left[\begin{array}{c}
\varepsilon_{11} \\
\vdots \\
\varepsilon_{15} \\
\varepsilon_{21} \\
\vdots \\
\varepsilon_{25}
\end{array}\right]
$$

Equation 7 can be written in regression model as follows:

$\lambda=X \beta+\varepsilon$

Next, we will be sought estimator to estimate a parameter $\beta$ in the above model by minimizing the error:

$$
S=\varepsilon^{* \prime} \varepsilon=\left(\lambda^{*}-X^{*} \beta\right)^{\prime}\left(\lambda^{*}-X^{*} \beta\right)
$$

Or:

$$
S=\lambda^{* \prime} \lambda^{*}-\lambda^{* \prime} X^{*} \beta-\beta^{\prime} X^{* \prime} \lambda^{*}+\beta^{\prime} X^{* \prime} X^{*} \beta
$$

That can be minimized by finding the first partial derivatives of the function $S$ against $\beta$ and equating to zero:

$$
\begin{aligned}
& \frac{\partial S}{\partial \beta}=-\lambda^{* \prime} X^{*}-\lambda^{* \prime} X^{*}+2 \hat{\beta}^{\prime} X^{* \prime} X^{*}=0 \\
& -2 \lambda^{* \prime} X^{*}+2 \hat{\beta}^{\prime} X^{* \prime} X^{*}=0
\end{aligned}
$$

We have:

$$
X^{* \prime} X^{*} \hat{\beta}=X^{* \prime} \lambda^{*}
$$

Besides that:

$$
\begin{aligned}
& (C X)^{\prime}(C X) \hat{\beta}=(C X)^{\prime}(C \lambda) \text { and } \\
& X^{\prime} C^{\prime} C X \hat{\beta}=X^{\prime} C^{\prime} C \lambda
\end{aligned}
$$

Because of $\Omega^{-1}=C^{\prime} C$, so Equation 8 can be written by:

$X^{\prime} \Omega^{-1} X \hat{\beta}=X^{\prime} \Omega^{-1} \lambda$

By multiply Equation 9 with $\left(X^{\prime} \Omega^{-1} X\right)^{-1}$, we have SUR estimator for $\beta$ as follows:

$$
\left(X^{\prime} \Omega^{-1} X\right)^{-1} X^{\prime} \Omega^{-1} X \hat{\beta}=\left(X^{\prime} \Omega^{-1} X\right)^{-1} X^{\prime} \Omega^{-1} \lambda
$$

We know that $\left(X^{\prime} \Omega^{-1} X\right)^{-1} X^{\prime} \Omega^{-1} X=I$, based on (10) we have:

$$
\begin{aligned}
& I \hat{\beta}=\left(X^{\prime} \Omega^{-1} X\right)^{-1} X^{\prime} \Omega^{-1} \lambda \text { or } \\
& \hat{\beta}^{S U R}=\left(X^{\prime} \Omega^{-1} X\right)^{-1} X^{\prime} \Omega^{-1} \lambda
\end{aligned}
$$


Vector of disturbances $\Omega$ in equation (10) is assumed as follows:

$$
\operatorname{Var}[\epsilon]=\operatorname{Var}\left[\left[\begin{array}{l}
\epsilon_{1} \\
\epsilon_{2}
\end{array}\right]\right],=E\left[\left(\left[\begin{array}{l}
\epsilon_{1} \\
\epsilon_{2}
\end{array}\right]-0\right)\left(\left[\begin{array}{l}
\epsilon_{1} \\
\epsilon_{2}
\end{array}\right]-0\right)^{\prime}\right]
$$

We know that $E\left[\left[\begin{array}{l}\epsilon_{1} \\ \epsilon_{2}\end{array}\right]\right]=0$, so that:

$$
\begin{aligned}
& \operatorname{Var}[\epsilon]=\left[\left[\begin{array}{ll}
\epsilon_{1} \epsilon_{1}^{\prime} & \epsilon_{1} \epsilon_{2}^{\prime} \\
\epsilon_{2} \epsilon_{1}^{\prime} & \epsilon_{2} \epsilon_{2}^{\prime}
\end{array}\right]\right], \\
& \left.=\left[\begin{array}{ll}
E\left[\epsilon_{1} \epsilon_{1}^{\prime}\right] & E\left[\epsilon_{1} \epsilon_{2}^{\prime}\right] \\
E\left[\epsilon_{2} \epsilon_{1}^{\prime}\right] & E\left[\epsilon_{2} \epsilon_{2}^{\prime}\right]
\end{array}\right]\right] \\
& =\left[\begin{array}{ll}
\operatorname{cov}\left(X_{1}, X_{1}\right) I_{n} & \operatorname{cov}\left(X_{1}, X_{2}\right) I_{n} \\
\operatorname{cov}\left(X_{2}, X_{1}\right) I_{n} & \operatorname{cov}\left(X_{2}, X_{2}\right) I_{n}
\end{array}\right] \\
& =\left[\begin{array}{ll}
\operatorname{cov}\left(X_{1}, X_{1}\right) & \operatorname{cov}\left(X_{1}, X_{2}\right) \\
\operatorname{cov}\left(X_{2}, X_{1}\right) & \operatorname{cov}\left(X_{2}, X_{2}\right)
\end{array}\right] \otimes I_{n}, \Omega=\sum \otimes I_{n}
\end{aligned}
$$

where, $I$ is identity $E\left(\epsilon_{n j} \in_{j t}\right)=\operatorname{cov}\left(X_{n}, X_{j}\right)$ for $t=1,2$. Inverse for Equation 11 as follows:

$$
\Omega^{-1}=\left[\begin{array}{ll}
\sigma^{11} I_{n-p} & \sigma^{12} I_{n-p} \\
\sigma^{21} I_{n-p} & \sigma^{22} I_{n-p}
\end{array}\right]=\sum^{-1} \otimes I_{n-p}
$$

So that estimator SUR for $\beta$ in Equation 10 can be denoted by:

$$
\hat{\beta}^{S U R}=\left(X^{\prime}(\Sigma)^{-1} X^{\prime}\right)^{-1} X^{\prime}(\Sigma \otimes I)^{-1} \lambda
$$

In case of variance-covariance matrix is unknown, so we have to estimate the value of entry matrix in vector disturbances $\Omega$ in Equation 10:

$$
\operatorname{Var}[\epsilon]=E\left[\left(\left[\begin{array}{l}
\hat{\epsilon}_{1} \\
\hat{\epsilon}_{2}
\end{array}\right]-0\right)\left(\left[\begin{array}{l}
\hat{\epsilon}_{1} \\
\hat{\epsilon}_{2}
\end{array}\right]-0\right)^{\prime}\right]
$$

Since $E\left[\left[\begin{array}{c}\hat{\epsilon}_{1} \\ \hat{\epsilon}_{2}\end{array}\right]\right]=0$ so that:

$$
\operatorname{Var}[\hat{\epsilon}]=\left[\left[\begin{array}{ll}
\hat{\epsilon}_{1} \hat{\epsilon}_{1}^{\prime} & \hat{\epsilon}_{1} \hat{\epsilon}_{2}^{\prime} \\
\hat{\epsilon}_{2} \hat{\epsilon}_{1}^{\prime} & \hat{\epsilon}_{2} \hat{\epsilon}_{2}^{\prime}
\end{array}\right]\right]
$$

Or:

$$
\begin{aligned}
& \operatorname{Var}[\hat{\epsilon}]=\left[\begin{array}{ll}
E\left[\hat{\epsilon}_{1} \hat{\epsilon}_{1}^{\prime}\right] & E\left[\hat{\epsilon}_{1} \hat{\epsilon}_{2}^{\prime}\right] \\
E\left[\hat{\epsilon}_{2} \hat{\epsilon}_{1}^{\prime}\right] & E\left[\hat{\epsilon}_{2} \hat{\epsilon}_{2}^{\prime}\right]
\end{array}\right] \\
& =\left[\begin{array}{ll}
\frac{1}{n} \operatorname{cov}\left(X_{1}, X_{1}\right) I_{n} & \frac{1}{n} \operatorname{cov}\left(X_{1}, X_{2}\right) I_{n} \\
\frac{1}{n} \operatorname{cov}\left(X_{2}, X_{1}\right) I_{n} & \frac{1}{n} \operatorname{cov}\left(X_{2}, X_{2}\right) I_{n}
\end{array}\right] \\
& =\left[\begin{array}{ll}
\frac{1}{n} \operatorname{cov}\left(X_{1}, X_{1}\right) I_{n} & \frac{1}{n} \operatorname{cov}\left(X_{1}, X_{2}\right) I_{n} \\
\frac{1}{n} \operatorname{cov}\left(X_{2}, X_{1}\right) I_{n} & \frac{1}{n} \operatorname{cov}\left(X_{2}, X_{2}\right) I_{n}
\end{array}\right] \otimes I_{n}
\end{aligned}
$$

where, $I$ is identity matrix and $E\left(\hat{\epsilon}_{n t} \hat{\epsilon}_{j t}\right)=\frac{1}{n} \operatorname{cov}\left(X_{n}, X_{j}\right)$ for $t=1,2 ; n$ is the number of data. Inverse of disturbances vector is:

$$
\Omega^{-1}=\left[\begin{array}{cc}
\hat{\sigma}^{11} I_{n} & \hat{\sigma}^{12} I_{n} \\
\hat{\sigma}^{21} I_{n} & \hat{\sigma}^{22} I_{n}
\end{array}\right]=\sum^{-1} \otimes I_{n}
$$

Thus, SUR estimator for variance covariance matrix that unknown is:

$$
\hat{\beta}^{S U R}=\left[\begin{array}{ll}
\hat{\sigma}^{11} X_{1}^{\prime} X_{1} & \hat{\sigma}^{12} X_{1}^{\prime} X_{2} \\
\hat{\sigma}^{21} X_{2}^{\prime} X_{1} & \hat{\sigma}^{22} X_{2}^{\prime} X_{2}
\end{array}\right]^{-1}\left[\begin{array}{l}
\hat{\sigma}^{11} X_{1}^{\prime} Y_{1}+\hat{\sigma}^{12} X_{1}^{\prime} Y_{2} \\
\hat{\sigma}^{21} X_{2}^{\prime} Y_{1}+\hat{\sigma}^{22} X_{2}^{\prime} Y_{2}
\end{array}\right]
$$

\section{Discussion}

Intensity of rainfall from January 2010 until December 2014 at both of Paotere and Mandai stations given in Table 1. In this table, we know that there are dry months for two stations during 2011 until 2015, such as June, July, August, September, October.

Generally, intensity of rainfall in June-October both on the Mandai and Paotere stations indicates a value of 0 . In other words there is no high rainfall in those months, so it cannot be used in modeling rainfall data. The data used in the modeling of precipitation data above is data during November-May. Correlation between the residual error in the first and second models on rainfall data is 0.85836 . It shows that this value indicates there is a high auto correlation between the two models so that the relationship between the residual correlation models could be improving the efficiency of estimation theoretically. Thus, parameter estimation using the SUR method appropriate to use this model. The results of parameter estimations for two models are given in Table 2. The negative sign on the auto correlation coefficients above shows the relation that is not the same direction for both models. In other words an increase in the value of the first model error will be accompanied by a decrease of error in the second model and vice versa. 
The Table 3 shows standard deviation and correlation of model using SUR method is better than OLS method. It can be seen that deviation standard of model using SUR method is less than SUR method.

Table 1. Intensity of rainfall in Paotere and Mandai Stations for 5 years

\begin{tabular}{|c|c|c|c|c|c|}
\hline \multicolumn{3}{|c|}{ Intensity (mm) } & \multicolumn{3}{|c|}{ Intensity (mm) } \\
\hline & Paotere & Mandai & & Paotere & Mandai \\
\hline \multirow[t]{5}{*}{ Jan } & 0.16129 & 0.22581 & July & 0.03226 & 0.03226 \\
\hline & 0.12903 & 0.12903 & & 0.00000 & 0.00000 \\
\hline & 0.12903 & 0.09677 & & 0.00000 & 0.00000 \\
\hline & 0.19354 & 0.29033 & & 0.00000 & 0.06452 \\
\hline & 0.09677 & 0.12903 & & 0.00000 & 0.00000 \\
\hline \multirow[t]{5}{*}{ Feb } & 0.07142 & 0.10714 & Aug & 0.03226 & 0.00000 \\
\hline & 0.07142 & 0.10714 & & 0.00000 & 0.00000 \\
\hline & 0.10345 & 0.06897 & & 0.00000 & 0.00000 \\
\hline & 0.07143 & 0.14286 & & 0.00000 & 0.00000 \\
\hline & 0.07143 & 0.10714 & & 0.00000 & 0.00000 \\
\hline \multirow[t]{5}{*}{ Mar } & 0.06452 & 0.00000 & Sept & 0.06667 & 0.06667 \\
\hline & 0.25806 & 0.03226 & & 0.00000 & 0.00000 \\
\hline & 0.22581 & 0.12903 & & 0.00000 & 0.00000 \\
\hline & 0.03226 & 0.03226 & & 0.00000 & 0.00000 \\
\hline & 0.03229 & 0.03226 & & 0.00000 & 0.00000 \\
\hline \multirow[t]{5}{*}{ Apr } & 0.03333 & 0.03333 & Okt & 0.03226 & 0.00000 \\
\hline & 0.10000 & 0.03333 & & 0.00000 & 0.00000 \\
\hline & 0.00000 & 0.03333 & & 0.00000 & 0.00000 \\
\hline & 0.10000 & 0.06667 & & 0.00000 & 0.00000 \\
\hline & 0.03333 & 0.06667 & & 0.00000 & 0.00000 \\
\hline \multirow[t]{5}{*}{ May } & 0.03226 & 0.03226 & Nov & 0.06667 & 0.03333 \\
\hline & 0.00000 & 0.00000 & & 0.03333 & 0.06667 \\
\hline & 0.03226 & 0.03226 & & 0.00000 & 0.00000 \\
\hline & 0.03226 & 0.00000 & & 0.03333 & 0.06667 \\
\hline & 0.00000 & 0.00000 & & 0.03333 & 0.00000 \\
\hline \multirow[t]{5}{*}{ June } & 0.03333 & 0.00000 & Dec & 0.16129 & 0.06452 \\
\hline & 0.00000 & 0.00000 & & 0.19354 & 0.16129 \\
\hline & 0.00000 & 0.00000 & & 0.09677 & 0.03226 \\
\hline & 0.03333 & 0.00000 & & 0.19355 & 0.29032 \\
\hline & 0.03333 & 0.00000 & & 0.12903 & 0.19355 \\
\hline
\end{tabular}

Table 2. The result of parameter estimation with SUR and OLS methods

\begin{tabular}{lrrr}
\hline$\beta^{\text {SUR }(1)}$ & $\beta^{\text {SUR }(2)}$ & $\beta^{\text {OLS }}(1)$ & $\beta^{\text {OLS }(2)}$ \\
\hline-0.04877 & -0.01819 & 0.20024 & 0.01922 \\
0.51183 & 0.99887 & -0.00051 & 0.16597 \\
0.06288 & 0.07436 & 0.04264 & 0.13998 \\
-0.21569 & -0.05627 & 0.37745 & 0.18283 \\
1.26147 & -0.39065 & -0.84429 & -0.22967 \\
0.10601 & -0.00936 & 0.14318 & 0.13083 \\
0.56294 & 1.90591 & 0.00248 & 0.08867 \\
0.62263 & 1.48729 & -0.01293 & -0.08329 \\
\hline
\end{tabular}

Table 3. Deviation Standard and Correlation for SUR and OLS methods

\begin{tabular}{lllll}
\hline & SUR(1) & SUR(2) & OLS(1) & OLS(2) \\
\hline $\begin{array}{l}\text { Deviation } \\
\text { standard }\end{array}$ & 0.02107 & 0.02424 & 0.06875 & 0.02569 \\
Correlation & 0.98831 & 0.99182 & 0.91785 & 0.99628 \\
\hline
\end{tabular}

\section{Conclusion}

Modeling of rainfall intensity into a spatial point process models using data from two adjacent stations can be denoted as a system represented by the Seemingly Unrelated Regression Equation model. This is done with the assumption that there is a residual correlation between adjacent locations. Results of the parameter estimation of the model for two selected stations (Paotere and Mandai) showed that the SUR method is better than the OLS method. Therefore, SUR method is more representative than OLS method to estimate parameters model of rainfall for both of Paotere and Mandai stations. This is because both of the monitoring stations have a location that is not far apart.

\section{Acknowledgement}

The authors thank to Mr. Nurdin who provided copy editing in this article. Our thank to the referee for helpful suggestions on the presentation of the results and for bringing recent references to our attention.

\section{Funding Information}

This research is supported by competitive research grant "Penelitian Unggulan Perguruan Tinggi 2015", ministry of research, technology and higher education, Indonesia.

\section{Author's Contributions}

Nurtiti Sunusi: Participated in designing a research mechanism scheme from beginning to end, participated in writing of the manuscript, conduct studies literature, Assessing and estimating model parameters.

Erna Tri Herdiani: Participated in analyzing data, conducting data simulation and Perform proof reading.

Fachrul Nawawi: Perform recording of data.

Giarno: Participated in perform collection data and typing articles.

\section{Ethics}

This article is original and contains unpublished material. The corresponding author confirms that all of the other authors have read and approved the manuscript and no ethical issues involved.

\section{References}

Alaba, O.O., E.O. Olubusoye and S.O. Ojo, 2010 Efficiency of seemingly unrelated regression estimator over the ordinary least square. Eur. J. Scientific Res., 39: $153-160$.

Atanlogun, S.K., O.A. Edwin and Y.O. Afolabi, 2014. On comparative modeling of GLS and OLS estimating techniques. Int. J. Scientific Technol. Res., 3: 125-128. 
Cameron, D., K. Beven and J. Tawn, 2000. An evaluation of three stochastic rainfall models. J. Hydrol., 228: 130-149. DOI: 10.1016/S0022-1694(00)00143-8

Daley, D.J. and D. Vere Jones, 2013. An Introduction to the Theory of Point Processes. Springer Science and Business Media, New York, ISBN-10: 1475720017, pp: 702.

Hill, C.R., W.E. Griffiths and G.C. Lim, 2011. Principles of Econometrics. 4th Edn., John Wiley and Sons, Hoboken, Inc., ISBN-10: 0470626739, pp: 784.

Onof, C., R. Chandler, A. Kakou, P. Northrop and H. Wheater et al., 2000. Rainfall modelling using Poisson-cluster processes: A review of developments. Stochastic Environ. Res. Risk Assessment, 14: 384-411. DOI: $10.1007 /$ s004770000043
Rodriguez-Iturbe, I., D.R. Cox and V. Isham, 1987. Some models for rainfall based on stochastic point processes. Proc. R. Soc. Lond. A, 410: 269-288. DOI: 10.1098/rspa.1987.0039

Vasco, A.P., 2012. The Use of Seemingly Unrelated Regression (SUR) to predict the carcass composition of lambs. FOI working paper, Institute of Food and Resource Economics, University of Copenhagen. 